\title{
Mobile Health Strategies to Tackle Skin Neglected Tropical Diseases With Recommendations From Innovative Experiences: Systematic Review
}

Carme Carrion ${ }^{1,2,3}$, MSc, PhD; Noemí Robles ${ }^{1,2,3}, \mathrm{PhD}$; Oriol Sola-Morales ${ }^{4}, \mathrm{MD}, \mathrm{PhD}$; Marta Aymerich ${ }^{1,2}, \mathrm{MD}, \mathrm{PhD}$, $\mathrm{MPH}$; Jose Antonio Ruiz Postigo ${ }^{5}, \mathrm{MD}, \mathrm{PhD}$

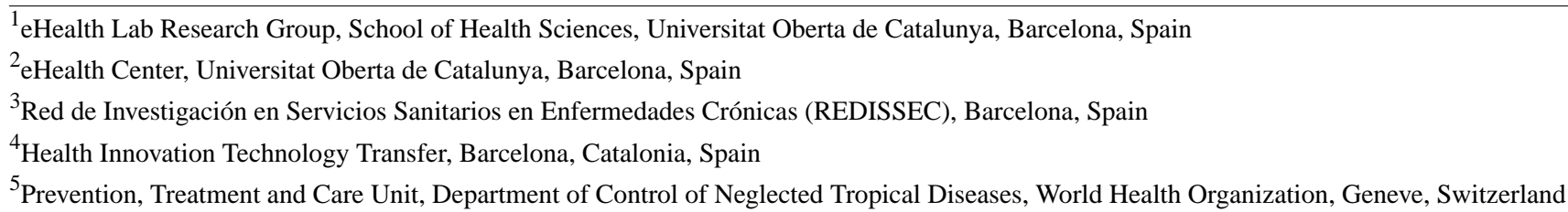

\section{Corresponding Author:}

Carme Carrion, MSc, PhD

eHealth Lab Research Group

School of Health Sciences

Universitat Oberta de Catalunya

Rambla Poble Nou 156

Barcelona, 08035

Spain

Phone: 3414163403200

Email: mcarrionr@uoc.edu

\section{Abstract}

Background: Neglected tropical diseases (NTDs) represent a diverse group of 20 communicable diseases that occur in tropical and subtropical areas in 149 countries, affecting over 1 billion people and costing developing economies billions of dollars every year. Within these diseases, those that present lesions on the skin surface are classified as skin NTDs (sNTDs). Mobile health interventions are currently being used worldwide to manage skin diseases and can be a good strategy in the epidemiological and clinical management of sNTDs.

Objective: We aimed to analyze existing evidence about mobile health interventions to control and manage sNTDs in low- and middle-income countries (LMICs) and make recommendations for what should be considered in future interventions.

Methods: A systematic review was conducted of the MEDLINE, Embase, and Scopus databases over 10 years up to April 30 , 2020. All types of clinical studies were considered. Data were synthesized into evidence tables. Apps were selected through a comprehensive systematic search in the Google Play Store and Apple App Store conducted between March 20 and April 15, 2020.

Results: From 133 potentially relevant publications, 13 studies met our criteria (9.8\%). These analyzed eight different interventions (three SMS text messaging interventions and five app interventions). Six of the 13 (46\%) studies were community-based cross-sectional studies intended to epidemiologically map a specific disease, mainly lymphatic filariasis, but also cutaneous leishmaniasis, leprosy, and NTDs, as well as sNTDs in general. Most of the studies were considered to have a high (5/13, 39\%) or moderate $(4 / 13,31 \%)$ risk of bias. Fifteen apps were identified in the Google Play Store, of which three were also in the Apple App Store. Most of the apps $(11 / 15,73 \%)$ were targeted at health care professionals, with only four targeted at patients. The apps focused on scabies $(3 / 15,20 \%)$, lymphatic filariasis $(3 / 15,20 \%)$, cutaneous leishmaniasis $(1 / 15,7 \%)$, leprosy $(1 / 15,7 \%)$, yaws and Buruli ulcer $(1 / 15,7 \%)$, tropical diseases including more than one sNTDs $(3 / 15,20 \%)$, and NTDs including sNTDs $(2 / 15$, $13 \%)$. Only 1 (7\%) app focused on the clinical management of sNTDs.

Conclusions: All mobile health interventions that were identified face technological, legal, final user, and organizational issues. There was a remarkable heterogeneity among studies, and the majority had methodological limitations that leave considerable room for improvement. Based on existing evidence, eight recommendations have been made for future interventions. 
(JMIR Mhealth Uhealth 2020;8(12):e22478) doi: 10.2196/22478

\section{KEYWORDS}

mHealth; mobile health; neglected tropical diseases; skin neglected tropical diseases; apps; SMS text messaging; low- and middle-income countries

\section{Introduction}

According to the World Health Organization (WHO), neglected tropical diseases (NTDs) represent a diverse group of 20 communicable diseases that occur in tropical and subtropical conditions in 149 countries, affecting over 1 billion people and costing developing economies billions of dollars every year [1]. These diseases include protozoal, bacterial, helminth, and viral infections [2] that cause vast suffering, stigma, and disability, and frequently lead to death [3]. As a result, NTDs trap impoverished people in a cycle of poverty and disease. Collectively, NTDs are among the most devastating of communicable diseases in terms of not only the global health burden (26.1 million disability-adjusted life-years) [3], but also the impact on development and overall economic productivity in low- and middle-income countries (LMICs).

The WHO has further categorized NTDs that primarily present as lesions on the skin (lumps or swelling, ulcers, swollen limbs, and patches on the face or body) into the skin NTD (sNTD) group. This includes Buruli ulcers, cutaneous leishmaniasis, post-kala-azar dermal leishmaniasis, leprosy, lymphatic filariasis (lymphoedema and hydrocele), mycetoma, onchocerciasis, fungal infections, scabies, and yaws. sNTDs not only cause considerable disability and stigma, but also exacerbate poverty [4-6]. The integration of mapping, surveillance, clinical diagnosis, and management has only been achieved in a limited range of settings and disease groupings [7]. Unfortunately, there has been relatively little investment in laboratory research, epidemiology, diagnostic tools, and management strategies to control tropical skin diseases. Visual examination provides an opportunity to screen people in communities or children in schools to identify multiple conditions in a single visit. This common approach to skin diseases justifies the integrated delivery of health care interventions to both increase cost-effectiveness and expand coverage [8,9].

Various innovative methods have been used to enhance the clinical management and epidemiological surveillance of, among others, skin and infectious diseases worldwide. These include, but are not limited to, technological methods such as telemedicine, artificial intelligence, and mobile health (mHealth) [10]. mHealth has been proven to be a promising tool to improve the diagnosis and treatment of several diseases such as skin cancer [11]. Telemedicine interventions have been implemented to perform teleconsultation and to improve the diagnosis and treatment of several diseases in countries with poor resources, and they have been proven to be effective in achieving its goal $[12,13]$. Teledermatological interventions, consisting of real-time videoconferencing or asynchronous transition of images between different dermatologists or between patients and clinicians, have been used for some years. Numerous studies have been published describing the potential of teledermatology in low-income settings, such as the review performed by
Médecins Sans Frontières to assess the tele-expertise system it had implemented to improve access to specialized clinical support for its field health workers [14]. These interventions are not yet scaled up, and although trials seem to confirm they are effective, they show some limitations that should be considered, such as the need for both a dermatologist available on demand and a stable and strong internet connection [15]. Artificial intelligence also holds promise in the diagnosis of skin conditions to a good degree of accuracy [16] and selecting the best treatment for a specific infectious disease [17]. However, these techniques are still under development and not yet available for implementation in the clinical management of sNTDs [18].

The WHO Global Observatory for eHealth defines mHealth as "medical and public health practice supported by mobile devices, such as mobile phones, patient monitoring devices, personal digital assistants, and other wireless devices" [19]. The prevention and management of chronic diseases through mHealth strategies has increased over recent years, mainly in high-income countries. However, there is still a lack of evidence for its efficacy, effectiveness, and safety [20]. Attempts have been made to improve the effective surveillance and control of infectious disease outbreaks [21], but very few interventions have been implemented in LMICs where sNTDs are endemic.

Given the high number of smartphone users worldwide (around 3 billion) [22] and the high penetration of smartphones in groups with low socioeconomic status, health-related mobile apps provide an opportunity to overcome traditional barriers to the control and clinical management of sNTDs in LMICs [23,24]. Nevertheless, the vast increase in low-cost health-related apps that are not regulated by health care policymakers raises important areas of concern, including quality, usability, and the need to educate consumers regarding the potentially beneficial (or harmful) content of apps [25]. Currently, of the over 325,000 health-related apps available [26], over 500 are skin related, with 90 providing self-surveillance and diagnosis [27]. Evaluation of six apps for skin cancer diagnosis has been conducted in high-income countries with discouraging results [28], and content analysis of 123 apps demonstrated that all were in need of improvement [29].

Therefore, it is clear that research on implementing mHealth strategies is required to improve the epidemiological surveillance and clinical management of sNTDs in LMICs. The main objective of this article is to analyze existing evidence about mHealth interventions to control and manage sNTDs in LMICs and make recommendations of what should be considered in future interventions. 


\section{Methods}

\section{Systematic Review}

\section{Information Sources}

Searches were conducted in the following databases: MEDLINE, Embase, and Scopus. This was complemented with a manual search of references in key journal archives. All published articles in the 10 years up to April 30, 2020, were considered with no restrictions on language. The reference lists of all selected studies were cross-checked for additional reports. A flow diagram of papers selected has been reported according to the Preferred Reporting Items for Systematic Reviews and Meta-Analyses (PRISMA) statement [30] (Figure 1).

Figure 1. PRISMA flow diagram of the selection of papers for inclusion in the review. NTD: neglected tropical disease.

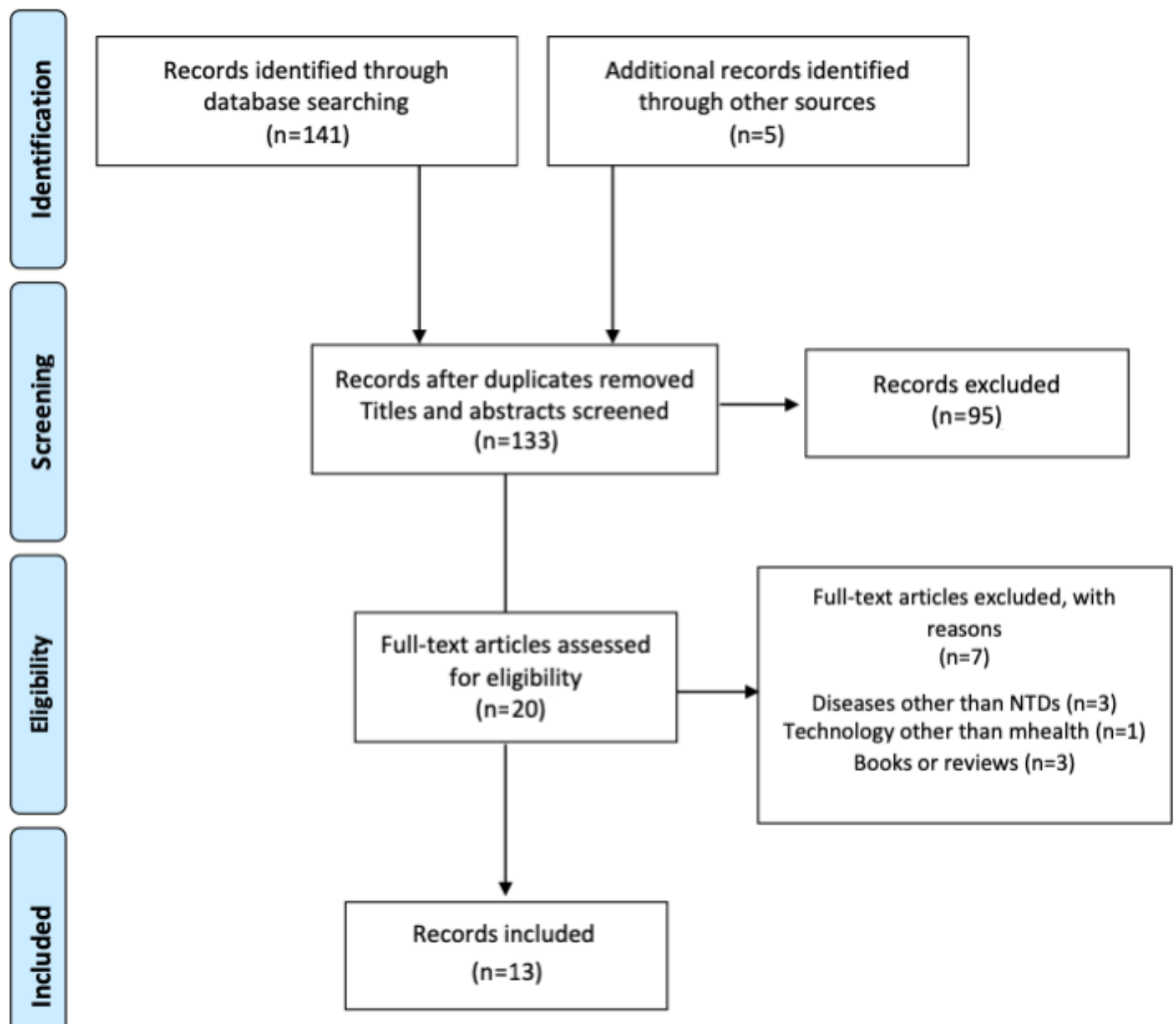

\section{Search Strategy}

The search strategy included both controlled vocabulary and free-text terms. The terms were smartphone, neglected tropical diseases, Buruli ulcer, fungal disease, leishmaniasis, leprosy, lymphatic filariasis, lymphoedema, mycetoma, onchocerciasis, scabies, yaws, mHealth, SMS, and apps (Multimedia Appendix $1)$.

\section{Eligibility Criteria}

The criteria for inclusion were reporting on mHealth diagnoses, treatment, epidemiological surveillance, and prevention of
sNTDs. All trial designs, regardless of risk of bias, were considered eligible. Animal studies were excluded. Letters, editorials, protocols, and reviews were not included.

\section{Study Selection and Data Collection Process}

All identified references were imported into Mendeley (v1.18), and duplicate references were eliminated. Articles that met the inclusion criteria were full-text reviewed by two independent reviewers. Any disagreements were resolved by a third reviewer. Study features and outcomes were entered into a database specifically designed for this review. Risk of bias was assessed 
according to Scottish Intercollegiate Guidelines Network (SIGN) codes for study assessment [31].

\section{App Selection}

\section{Search Strategy}

Apps were identified through a comprehensive systematic search of the Google Play Store and Apple App Store between March 20 and April 15, 2020, using 14 search terms in English and Spanish across all store categories (Multimedia Appendix 1).

\section{Screening Procedure}

Each term was searched separately in both app stores, with two researchers performing identical independent searches. Information in the app store description for each result was reviewed. The inclusion criteria were as follows: (1) the purpose should be diagnosis, treatment, prevention, patient empowerment, health literacy, or epidemiological surveillance; (2) the main disease should be one of the 10 sNTDs; and (3) the app should be available in English or Spanish. Apps were excluded if they did not have a clear focus on the epidemiological or clinical management of sNTDs and focused on other issues, such as conferences, atlases of skin diseases, and a general approach to tropical diseases. Apps that met the eligibility criteria were selected and their URLs were saved. After the independent search, the two researchers compared the lists of apps they identified as eligible and performed analyses. Light and early versions of the apps were excluded in favor of full late versions.

\section{Data Collection}

Information and features were extracted from app store descriptions and available screenshots. In cases where not enough information was provided, the apps were downloaded, installed, and tested in smartphones running Android or iOS operating system.

\section{Results}

\section{Selection of Studies}

A total of 133 potentially relevant publications were identified as eligible. After screening the titles and abstracts, 20 out of $133(15.0 \%)$ were accepted for full-text review. Out of these 20, 7 (35\%) were excluded for not meeting the inclusion criteria. They focused on diseases other than sNTDs $(n=3)$, used interventions without any mHealth element $(n=1)$, or were reviews or book chapters $(n=3)$. After peer-review, 13 articles were included in this nonquantitative review (Figure 1).

The main characteristics of the 13 studies included are detailed in Table 1. Studies appear in chronological order and then alphabetical order of the first author. The studies were highly heterogeneous in their design, resulting from their heterogeneous objectives. Six (46\%) studies [32-37] were reported to be community-based cross-sectional studies with the main objective of epidemiologically mapping a specific disease based on its clinical symptoms. Four out of $13(31 \%)$ studies had different designs as follows: a mixed-methods approach to define challenges to be considered [38]; different implementation scenarios for a specific mHealth intervention [39]; the sustainability of an intervention [40]; and the process of developing a specific mHealth tool or assessing its usability $[10,38]$. One trial was a cross-sectional study analyzing the accuracy of a new diagnosis strategy [41], and another was a prospective cohort study testing the efficacy of an mHealth strategy in training health care workers [42]. There was only one controlled nonrandomized study to test the effectiveness of an intervention [43]. Most of the studies had a high (5/13, $39 \%$ ) or moderate $(4 / 13,31 \%)$ risk of bias according to SIGN quality criteria. Only three of the included studies were considered to have a low risk of bias [33,35,37].

Most of the studies $(n=8)$ were focused on lymphatic filariasis alone or together with podoconiosis or onchocerciasis. These define strategies to estimate the prevalence of the diseases or the most common clinical manifestations (hydrocele or lymphoedema). There was one study about NTDs in general [40] and one about sNTDs [10]. Only two studies focused on sNTDs other than lymphatic filariasis. Of these, one was on cutaneous leishmaniasis [38] and one was on leprosy [43]. All studies were conducted in LMICs where sNTDs are endemic, mainly Africa $(n=7)$. Two study areas were in the American continent, and all others were in Africa and Asia. The studies took place in the following 13 different countries: Tanzania $(n=4)$, Ethiopia $(n=3)$, Bangladesh $(n=2)$, Malawi $(n=2)$, Brazil $(\mathrm{n}=1)$, Bolivia $(\mathrm{n}=1)$, Colombia $(\mathrm{n}=1)$, Ghana $(\mathrm{n}=1)$, Indonesia $(n=1)$, Nepal $(n=1)$, Nigeria $(n=1)$, Mozambique $(n=1)$, and Peru $(n=1)$. Seven of the studies analyzed interventions in both rural and urban areas, four in rural areas only, and two in urban areas. 
Table 1. Characteristics of the selected studies.

\begin{tabular}{|c|c|c|c|c|c|c|c|c|}
\hline $\begin{array}{l}\text { Refer- } \\
\text { ence }^{a}\end{array}$ & Study design & $\begin{array}{l}\text { Study popula- } \\
\text { tion }\end{array}$ & $\mathrm{NTD}^{\mathrm{b}}$ & Tool & Objective & Intervention & Outcomes & $\begin{array}{l}\text { Risk of } \\
\text { bias }\end{array}$ \\
\hline $\begin{array}{l}\text { Sime } \\
2014 \text { [32] }\end{array}$ & $\begin{array}{l}\text { Community- } \\
\text { based cross- } \\
\text { sectional } \\
\text { study }\end{array}$ & $\begin{array}{l}\text { Ethiopia (659 } \\
\text { districts) } \\
\mathrm{n}=129,959 \\
\text { Urban and rural } \\
\text { areas }\end{array}$ & $\begin{array}{l}\mathrm{LF}^{\mathrm{c}} \\
\text { and } \\
\mathrm{PDC}^{\mathrm{d}}\end{array}$ & $\begin{array}{l}\text { LINKS } \\
\text { system }\end{array}$ & $\begin{array}{l}\text { To perform inte- } \\
\text { grated mapping } \\
\text { of the two dis- } \\
\text { eases }\end{array}$ & $\begin{array}{l}\text { Immunochromatographic } \\
\text { card tests of blood samples } \\
\text { to detect circulating } \\
\text { Wuchereria bancrofti. } \\
\text { Data collection through } \\
\text { questionnaires on a smart- } \\
\text { phone (GPS function in- } \\
\text { cluded), and data sent to a } \\
\text { cloud server for analysis. }\end{array}$ & $\begin{array}{l}\text { Real-time data collection. } \\
\text { Prevalence of lymphoede- } \\
\text { ma }(\mathrm{n}=8,110) \text { and } \\
\text { Wuchereria bancrofti } \\
(\mathrm{n}=139) \text {. } \\
\text { Budget halved with smart- } \\
\text { phone-based data collec- } \\
\text { tion. }\end{array}$ & $\begin{array}{l}\text { Moderate } \\
\text { Selection } \\
\text { bias }\end{array}$ \\
\hline $\begin{array}{l}\text { Luz } 2015 \\
{[40]}\end{array}$ & $\begin{array}{l}\text { Mixed-meth- } \\
\text { ods qualita- } \\
\text { tive study }\end{array}$ & $\begin{array}{l}\text { Southwestern } \\
\text { Amazon region } \\
\text { (Brazil, Perú, } \\
\text { and Bolivia) } \\
\mathrm{n}=47 \text { (15 medi- } \\
\text { cal doctors, } 15 \\
\text { researchers, } 13 \\
\text { nurses, and four } \\
\text { staff) } \\
\text { Rural area }\end{array}$ & NTD & $\begin{array}{l}\mathrm{Nu}- \\
\text { case }\end{array}$ & $\begin{array}{l}\text { To explore the } \\
\text { sustainability of } \\
\text { the intervention }\end{array}$ & $\begin{array}{l}\text { Design of Nu-case proto- } \\
\text { type. } \\
\text { Surveys, questionnaires, } \\
\text { sketching, and storyboard- } \\
\text { ing to define actors, tasks, } \\
\text { needs, and possible design. }\end{array}$ & $\begin{array}{l}\text { High perceived potential } \\
\text { of Nu-case in diagnosis, } \\
\text { disease monitoring and } \\
\text { surveillance, medical } \\
\text { records, case notification, } \\
\text { and medical research. } \\
\text { Low perceived potential in } \\
\text { health care management, } \\
\text { assisting diagnosis for } \\
\text { HCPs }{ }^{\mathrm{e}} \text { other than medical } \\
\text { doctors, and HCP training } \\
\text { tools. }\end{array}$ & $\begin{array}{l}\text { High } \\
\text { Clustered } \\
\text { data anal- } \\
\text { ysis with } \\
\text { a very } \\
\text { small } \\
\text { sample } \\
\text { size. }\end{array}$ \\
\hline $\begin{array}{l}\text { Stanton } \\
2015 \text { [33] }\end{array}$ & $\begin{array}{l}\text { Community- } \\
\text { based cross- } \\
\text { sectional sur- } \\
\text { vey }\end{array}$ & $\begin{array}{l}\text { Malawi (Chik- } \\
\text { wawa district) } \\
\mathrm{n}=107,331 \\
\text { Ghana (Ahanta } \\
\text { West district) } \\
\mathrm{n}=45,402 \\
\text { Rural area }\end{array}$ & $\mathrm{LF}$ & $\begin{array}{l}\text { Mea- } \\
\text { sure } \\
\text { SMS }\end{array}$ & $\begin{array}{l}\text { To map clinical } \\
\text { manifestation of } \\
L F\left(\operatorname{LE}^{\mathrm{f}} \text { and }\right. \\
\left.\mathrm{HYC}^{\mathrm{g}}\right) \\
\text { To pilot the } \\
\text { Measure SMS } \\
\text { tool }\end{array}$ & $\begin{array}{l}\text { HSAs }^{\mathrm{h}} \text { in Malawi and } \\
\text { CHWs } \\
\text { individual LE and HYC } \\
\text { case data. }\end{array}$ & $\begin{array}{l}\text { Ghana: prevalence of } 17.7 \\
\text { (LE) and } 33.0 \text { (HYC) per } \\
10,000 . \\
\text { Malawi: prevalence of } \\
76.9 \text { (LE) and } 70.5 \text { (HYC) } \\
\text { per 10,000. } \\
17 \% \text { of SMS messages in } \\
\text { Malawi and } 16 \% \text { in Ghana } \\
\text { contained an error ( } 41 \% \\
\text { were easy to solve). }\end{array}$ & Low \\
\hline $\begin{array}{l}\text { Mableson } \\
2017 \text { [39] }\end{array}$ & $\begin{array}{l}\text { Implementa- } \\
\text { tion study }\end{array}$ & $\begin{array}{l}\text { Ethiopia, } \\
\text { Malawi, Tanza- } \\
\text { nia, Nepal, and } \\
\text { Bangladesh } \\
\mathrm{n}=22 \text { million } \\
\text { people } \\
\text { Urban and rural } \\
\text { areas }\end{array}$ & $\mathrm{LF}$ & $\begin{array}{l}\text { Mea- } \\
\text { sure } \\
\text { SMS- } \\
\text { mor- } \\
\text { bidity }\end{array}$ & $\begin{array}{l}\text { To define imple- } \\
\text { mentation sce- } \\
\text { narios for LF } \\
\text { mapping }\end{array}$ & $\begin{array}{l}\text { Health workers collect data } \\
\text { in a traditional paper-based } \\
\text { survey and then send an } \\
\text { SMS (two tier) or directly } \\
\text { send an SMS (one tier) to } \\
\text { a central smartphone con- } \\
\text { nected to a cloud server. }\end{array}$ & $\begin{array}{l}\text { Four potential implementa- } \\
\text { tion reporting scenarios: } \\
\text { (1) urban, high-endemic } \\
\text { setting, two tier } \\
\text { (2) rural, high-endemic } \\
\text { setting, one tier } \\
\text { (3) rural, high-endemic } \\
\text { setting, two tier } \\
\text { (4) urban and rural, low- } \\
\text { endemic setting, one tier }\end{array}$ & $\begin{array}{l}\text { High } \\
\text { No } \\
\text { method- } \\
\text { ological } \\
\text { details }\end{array}$ \\
\hline $\begin{array}{l}\text { Mwingira } \\
2017 \text { [34] }\end{array}$ & $\begin{array}{l}\text { Community- } \\
\text { based cross- } \\
\text { sectional sur- } \\
\text { vey }\end{array}$ & $\begin{array}{l}\text { Tanzania (Mt- } \\
\text { wara Municipal } \\
\text { Council) } \\
\text { n=108,299 } \\
\text { Mainly urban } \\
\text { areas }\end{array}$ & LF & $\begin{array}{l}\text { SMS } \\
\text { through } \\
\text { the } \\
\text { GeoPoll } \\
\text { plat- } \\
\text { form }\end{array}$ & $\begin{array}{l}\text { To estimate } \\
\text { prevalences of } \\
\text { HYC and LE }\end{array}$ & $\begin{array}{l}\text { Interactive survey via SMS } \\
\text { to randomized selected } \\
\text { users. }\end{array}$ & $\begin{array}{l}\text { Response ratio of } 15.2 \% ; \\
\mathrm{n}=492(78 \% \text { male); mean } \\
\text { age } 20.1(\mathrm{SD} 6.5) \text { years } \\
\text { LE signs }=22.2 \%(95 \% \mathrm{CI} \\
17.4-24.8) \\
\text { HYC signs }=20.6 \%(95 \% \\
\text { CI 16.6-25.0) }\end{array}$ & $\begin{array}{l}\text { High } \\
\text { Self-re- } \\
\text { ported da- } \\
\text { ta } \\
\text { Incen- } \\
\text { tives giv- } \\
\text { en to par- } \\
\text { ticipants } \\
\text { Gender } \\
\text { and age } \\
\text { biased }\end{array}$ \\
\hline
\end{tabular}




\begin{tabular}{|c|c|c|c|c|c|c|c|c|}
\hline $\begin{array}{l}\text { Refer- } \\
\text { ence }^{a}\end{array}$ & Study design & $\begin{array}{l}\text { Study popula- } \\
\text { tion }\end{array}$ & $\mathrm{NTD}^{\mathrm{b}}$ & Tool & Objective & Intervention & Outcomes & $\begin{array}{l}\text { Risk of } \\
\text { bias }\end{array}$ \\
\hline $\begin{array}{l}\text { Mwingira } \\
2017 \text { [35] }\end{array}$ & $\begin{array}{l}\text { Community- } \\
\text { based cross- } \\
\text { sectional sur- } \\
\text { vey }\end{array}$ & $\begin{array}{l}\text { Tanzania (Dar } \\
\text { es Salaam) } \\
\mathrm{n}=5 \text { million } \\
\text { Urban area }\end{array}$ & $\mathrm{LF}$ & $\begin{array}{l}\text { Mea- } \\
\text { sure } \\
\text { SMS- } \\
\text { mor- } \\
\text { bidity } \\
\text { tool }\end{array}$ & $\begin{array}{l}\text { To locate LF } \\
\text { patients and esti- } \\
\text { mate prevalence }\end{array}$ & $\begin{array}{l}\text { SMS survey in a three- } \\
\text { phase intervention: paper- } \\
\text { based data collection } \\
\text { checked by a supervisor } \\
\text { and sent by SMS to a local } \\
\text { server. Data checked and } \\
\text { sent to a cloud-based serv- } \\
\text { er. }\end{array}$ & $\begin{array}{l}\text { Prevalence of } 133.6 \text { per } \\
10,000 ; n=6.889 \text {. } \\
\text { Less than } 20 \% \text { of SMS } \\
\text { messages had formatting } \\
\text { errors. } \\
\text { More than } 95 \% \text { of SMS } \\
\text { messages were sent to the } \\
\text { cloud server. }\end{array}$ & $\begin{array}{l}\text { Low } \\
\text { Some im- } \\
\text { plementa- } \\
\text { tion chal- } \\
\text { lenges }\end{array}$ \\
\hline $\begin{array}{l}\text { Pedram } \\
2017 \text { [41] }\end{array}$ & $\begin{array}{l}\text { Cross-sec- } \\
\text { tional study }\end{array}$ & $\begin{array}{l}\text { Control }(\mathrm{n}=77) \\
\text { and infected } \\
(\mathrm{n}=313) \\
\text { Various coun- } \\
\text { tries in Africa, } \\
\text { Asia, and } \\
\text { America } \\
\text { Rural and urban } \\
\text { areas }\end{array}$ & $\begin{array}{l}\mathrm{LF} \\
\text { and } \\
\mathrm{ONC}^{\mathrm{j}}\end{array}$ & $\begin{array}{l}\text { Smart- } \\
\text { phone- } \\
\text { based } \\
\text { micro- } \\
\text { scope }\end{array}$ & $\begin{array}{l}\text { To assess the } \\
\text { accuracy of a } \\
\text { new diagnostic } \\
\text { test }\end{array}$ & $\begin{array}{l}\text { All samples tested with the } \\
\text { new test including a } \\
\text { portable smartphone-based } \\
\text { microscope. }\end{array}$ & $\begin{array}{l}\text { Sensitivity of } 71 \% \text { and } \\
\text { specificity around } 100 \% \\
\text { for all tested species. }\end{array}$ & $\begin{array}{l}\text { Moderate } \\
\text { Informa- } \\
\text { tion bias }\end{array}$ \\
\hline $\begin{array}{l}\text { Karim } \\
2018 \text { [36] }\end{array}$ & $\begin{array}{l}\text { Community- } \\
\text { based cross- } \\
\text { sectional sur- } \\
\text { vey }\end{array}$ & $\begin{array}{l}\text { Bangladesh } \\
\text { (high and low } \\
\text { endemic dis- } \\
\text { tricts) } \\
\mathrm{n}=65 \text { million } \\
\text { people at risk } \\
\text { Rural and urban } \\
\text { areas }\end{array}$ & $\mathrm{LF}$ & $\begin{array}{l}\text { Mea- } \\
\text { sure } \\
\text { SMS }\end{array}$ & $\begin{array}{l}\text { To determine } \\
\text { HYC and LE } \\
\text { prevalence and } \\
\text { develop clinical } \\
\text { risk maps for } \\
\text { targeted inter- } \\
\text { ventions }\end{array}$ & $\begin{array}{l}\text { Paper-based census, active } \\
\text { case findings, and summa- } \\
\text { ry data per patient sent } \\
\text { through the system to a } \\
\text { central database. } \\
\text { In low endemic districts, } \\
\text { case findings via health fa- } \\
\text { cility data and confirmed } \\
\text { by mHealth }{ }^{\mathrm{k}} \text { trained profes- } \\
\text { sionals sending SMS. }\end{array}$ & $\begin{array}{l}\text { Prevalence of } 125.4 \text { per } \\
100,000 \text { in high-endemic } \\
\text { districts and } 2.4 \text { per } \\
100,000 \text { in low-endemic } \\
\text { districts. }\end{array}$ & $\begin{array}{l}\text { Moderate } \\
\text { Different } \\
\text { methods } \\
\text { used } \\
\text { mHealth } \\
\text { strategy } \\
\text { not fully } \\
\text { described } \\
\text { and vali- } \\
\text { dated }\end{array}$ \\
\hline $\begin{array}{l}\text { Martin- } \\
\text { dale } 2018 \\
{[37]}\end{array}$ & $\begin{array}{l}\text { Community- } \\
\text { based } \\
\text { cross-section- } \\
\text { al study }\end{array}$ & $\begin{array}{l}\text { Ethiopia (two } \\
\text { districts: } \\
\text { Hawella Tula } \\
\text { and Besa) } \\
\mathrm{n}=460,722 \\
\text { Rural area }\end{array}$ & $\begin{array}{l}\mathrm{LF} \\
\text { and } \\
\mathrm{PDC}\end{array}$ & $\begin{array}{l}\text { Mea- } \\
\text { sure } \\
\text { SMS- } \\
\text { mor- } \\
\text { bidity } \\
\text { tool }\end{array}$ & $\begin{array}{l}\text { To pilot feasibil- } \\
\text { ity and utility } \\
\text { for an integrat- } \\
\text { ed mapping of } \\
\text { the two diseases }\end{array}$ & $\begin{array}{l}\text { Survey conducted by } \\
\text { health extension workers } \\
(\mathrm{n}=59) \text {. } \\
\text { Clinical cases reported by } \\
\text { paper-based standard } \\
\text { forms and by SMS. }\end{array}$ & $\begin{array}{l}\text { Paper-based cases reported } \\
(\mathrm{n}=2.377) \\
\text { SMS method cases report- } \\
\text { ed }(\mathrm{n}=2.372) \\
P \text { value of } .94 \\
\text { Prevalence of } 64 \text { per } \\
10,000 . \\
\text { Cost saving }(13.7 \%) \text { with } \\
\text { the SMS method. }\end{array}$ & $\begin{array}{l}\text { Low } \\
\text { Potential } \\
\text { informa- } \\
\text { tion bias } \\
\text { in the real } \\
\text { condition }\end{array}$ \\
\hline $\begin{array}{l}\text { Mieras } \\
2018 \text { [10] }\end{array}$ & $\begin{array}{l}\text { Develop- } \\
\text { ment and pi- } \\
\text { lot study }\end{array}$ & $\begin{array}{l}\text { HCP in Nigeria } \\
\text { and in Mozam- } \\
\text { bique } \\
\text { Rural and urban } \\
\text { areas }\end{array}$ & sNTDs & $\begin{array}{l}\text { Ski- } \\
\text { nApp }\end{array}$ & $\begin{array}{l}\text { To support HCP } \\
\text { in the early diag- } \\
\text { nosis of sNTDs } \\
\text { in low resource } \\
\text { settings }\end{array}$ & $\begin{array}{l}\text { Development of SkinApp } \\
\text { with } 29 \text { diseases included } \\
\text { (six sNTDs) and assess- } \\
\text { ment of user friendliness } \\
\text { (semistructured interviews } \\
\text { and focus groups). } \\
\text { Implementation study }\end{array}$ & $\begin{array}{l}\text { SkinApp is considered a } \\
\text { good decision support sys- } \\
\text { tem. }\end{array}$ & $\begin{array}{l}\text { High } \\
\text { No } \\
\text { method- } \\
\text { ological } \\
\text { details }\end{array}$ \\
\hline $\begin{array}{l}\text { Navarro } \\
2018 \text { [38] }\end{array}$ & $\begin{array}{l}\text { Consensus } \\
\text { report }\end{array}$ & $\begin{array}{l}\text { Colombia (Tu- } \\
\text { maco) } \\
\text { Rural area }\end{array}$ & $\mathrm{CL}^{1}$ & $\begin{array}{l}\text { Guaral } \\
\text { App }\end{array}$ & $\begin{array}{l}\text { To define chal- } \\
\text { lenges and requi- } \\
\text { sites of mHealth } \\
\text { tools }\end{array}$ & $\begin{array}{l}\text { Development of Guaral } \\
\text { App to be adopted by vol- } \\
\text { unteer community workers } \\
\text { for diagnosis and mapping } \\
\text { of patients with CL. }\end{array}$ & $\begin{array}{l}\text { Key aspects are: } \\
\text {-Sociotechnical context } \\
\text {-Systems analysis } \\
\text {-Human-centered design }\end{array}$ & $\begin{array}{l}\text { Not appli- } \\
\text { cable }\end{array}$ \\
\hline $\begin{array}{l}\text { Akoko } \\
2019 \text { [42] }\end{array}$ & $\begin{array}{l}\text { Prospective } \\
\text { cohort study }\end{array}$ & $\begin{array}{l}\text { Tanzania (Mt- } \\
\text { wara and Lindi } \\
\text { regions) } \\
\text { Rural and urban } \\
\text { areas }\end{array}$ & HYC & $\begin{array}{l}\text { What- } \\
\text { sApp } \\
\text { plat- } \\
\text { form }\end{array}$ & $\begin{array}{l}\text { To test the effi- } \\
\text { cacy of a mo- } \\
\text { bile platform as } \\
\text { an adjunct in } \\
\text { supervision and } \\
\text { support for non- } \\
\text { surgical clini- } \\
\text { cians when } \\
\text { practicing hy- } \\
\text { drocelectomy }\end{array}$ & $\begin{array}{l}\text { Didactic and practical } \\
\text { training conducted by two } \\
\text { experts. } \\
\text { Photographs shared though } \\
\text { the WhatsApp platform for } \\
\text { group discussion, final ap- } \\
\text { proval of surgery, on table } \\
\text { and postoperative compli- } \\
\text { cations. }\end{array}$ & $\begin{array}{l}\text { Fifteen NPCs }{ }^{\mathrm{m}} \text { trained and } \\
\text { able to perform } 1337 \text { hy- } \\
\text { drocelectomies in } 1250 \\
\text { patients. } \\
\text { Mean procedure duration } \\
\text { of } 50.2 \text { min (SD } 0.24 \text { ) } \\
\text { Complication rate }<2.16 \%\end{array}$ & $\begin{array}{l}\text { Moderate } \\
\text { Small } \\
\text { sample of } \\
\text { NPCs } \\
\text { Selection } \\
\text { bias }\end{array}$ \\
\hline
\end{tabular}




\begin{tabular}{|c|c|c|c|c|c|c|c|c|}
\hline $\begin{array}{l}\text { Refer- } \\
\text { ence }^{a}\end{array}$ & Study design & $\begin{array}{l}\text { Study popula- } \\
\text { tion }\end{array}$ & $\mathrm{NTD}^{\mathrm{b}}$ & Tool & Objective & Intervention & Outcomes & $\begin{array}{l}\text { Risk of } \\
\text { bias }\end{array}$ \\
\hline \multirow{5}{*}{$\begin{array}{l}\text { Rach- } \\
\text { mani } \\
2019[43]\end{array}$} & \multirow{5}{*}{$\begin{array}{l}\text { Nonrandom- } \\
\text { ized con- } \\
\text { trolled longi- } \\
\text { tudinal obser- } \\
\text { vational } \\
\text { study }\end{array}$} & Indonesia & \multirow{5}{*}{$\begin{array}{l}\text { Lep- } \\
\text { rosy }\end{array}$} & \multirow{5}{*}{$\begin{array}{l}\text { e-lep- } \\
\text { rosy } \\
\text { frame- } \\
\text { work }\end{array}$} & \multirow{5}{*}{$\begin{array}{l}\text { To evaluate the } \\
\text { effectiveness of } \\
\text { an e-leprosy } \\
\text { framework in } \\
\text { PHC }^{\text {n }}\end{array}$} & \multirow{5}{*}{$\begin{array}{l}\text { Implementation of an e- } \\
\text { leprosy framework for pri- } \\
\text { mary health care for } 19 \\
\text { months. }\end{array}$} & \multirow{5}{*}{$\begin{array}{l}\text { Abandon rate of } 21 \% \text {. } \\
\text { Increase of } 21 \% \text { in on-time } \\
\text { completion and } 14.6 \% \text { in } \\
\text { attendance rates. }\end{array}$} & High \\
\hline & & $\begin{array}{l}\text { (Pekalongan } \\
\text { District Java) }\end{array}$ & & & & & & \multirow{4}{*}{$\begin{array}{l}\begin{array}{l}\text { Nonran- } \\
\text { domiza- } \\
\text { tion }\end{array} \\
\text { Sample } \\
\text { selection } \\
\text { bias }\end{array}$} \\
\hline & & $\mathrm{n}=124$ control, & & & & & & \\
\hline & & $\begin{array}{l}\mathrm{n}=64 \text { interven- } \\
\text { tion group }\end{array}$ & & & & & & \\
\hline & & $\begin{array}{l}\text { Rural and urban } \\
\text { areas }\end{array}$ & & & & & & \\
\hline
\end{tabular}

${ }^{\mathrm{a}}$ Studies are in chronological order and then alphabetical order of first author.

${ }^{\mathrm{b}} \mathrm{NTD}$ : neglected tropical disease.

${ }^{c}$ LF: lymphatic filariasis.

${ }^{\mathrm{d}}$ PDC: podoconiosis.

${ }^{\mathrm{e}} \mathrm{HCP}$ : health care professional.

${ }^{\mathrm{f}} \mathrm{LE}$ : lymphoedema.

${ }^{\mathrm{g}} \mathrm{HYC}$ : hydrocele.

${ }^{\mathrm{h}} \mathrm{HSA}$ : salaried health surveillance assistant.

${ }^{\mathrm{i}} \mathrm{CHW}$ : community health worker.

${ }^{\mathrm{j}} \mathrm{ONC}$ : onchocerciasis.

${ }^{\mathrm{k}}$ mHealth: mobile health.

${ }^{1} \mathrm{CL}$ : cutaneous leishmaniasis.

$\mathrm{m}_{\mathrm{NPC}}$ : nonphysician clinician.

${ }^{\mathrm{n}} \mathrm{PHC}$ : primary healthcare.

\section{Elements Included in the mHealth Interventions}

Only eight different mHealth interventions were identified (Table 2). Three were based on sending SMS text messages between health workers and a central web system $[33-35,37,39,41,43]$. Text messaging is an easy technology to use and, as these studies show, is useful for motivating patients to complete treatments. Health care services can be equipped with systems providing automated text message reminders for disease control [43]. The Measure SMS and Measure SMS-morbidity strategies have been tested and implemented in several different contexts. The ability to view and assess the quality of patient identification data in real time is a great asset. Enabling information on morbidity burden to be known almost instantaneously, as opposed to having to wait for the collation and digitization of paper forms, also facilitates more efficient provision of necessary care [35]. 
Table 2. Elements and challenges described in the mobile health interventions of the selected studies.

\begin{tabular}{|c|c|c|c|c|c|c|}
\hline \multirow[t]{2}{*}{ Tool Name } & \multicolumn{4}{|c|}{ Elements included } & \multirow[t]{2}{*}{ Challenges identified } & \multirow[t]{2}{*}{ References } \\
\hline & SMS & Web & App & $\begin{array}{l}\text { Cloud } \\
\text { server }\end{array}$ & & \\
\hline e-Leprosy framework & + & + & - & - & $\begin{array}{l}\text { - } \quad \text { Cost-benefit analysis } \\
\text { - } \quad \text { Need for a comprehensive strategy } \\
\text { - } \quad \text { Perceived usefulness of the technology } \\
\text { - } \quad \text { Perceived ease of use of the technology } \\
\text { - No internet connection in remote rural areas }\end{array}$ & Rachmani 2019 [43] \\
\hline GeoPoll and SMS & + & + & - & - & No challenges identified in the study & Mwingira 2017 [34] \\
\hline Guaral App & - & - & + & + & $\begin{array}{ll}\text { - } & \text { Sociotechnical context } \\
\text { - } & \text { Human-centered design } \\
\text { - } & \text { Iterative design } \\
\text { - } & \text { Usability } \\
\text { - } & \text { Technical concerns }\end{array}$ & Navarro 2018 [38] \\
\hline LINKS system & - & - & + & + & $\begin{array}{l}\text { - } \quad \text { Data ownership } \\
\text { - } \quad \text { Lack of technical expertise } \\
\text { - } \quad \text { Cost of smartphones } \\
\text { - } \quad \mathrm{HCP}^{\mathrm{a}} \text { perceptions about digital data collection } \\
\text { - } \quad \text { Batteries running out of charge } \\
\text { Lack of network }\end{array}$ & Sime 2014 [32] \\
\hline $\begin{array}{l}\text { Measure SMS and Measure } \\
\text { SMS-morbidity }\end{array}$ & + & + & - & + & $\begin{array}{ll}\text { - } & \text { Data accuracy } \\
\text { - } & \text { HCP training } \\
\text { - } & \text { Technological barriers } \\
\text { - } & \text { Cost } \\
-\quad \text { Poor network coverage } \\
\text { - } & \text { Battery supply } \\
\text { - } & \text { Heasibility } \\
& \text { change }\end{array}$ & $\begin{array}{l}\text { Stanton } 2015[33] \\
\text { Karim } 2019[36] \\
\text { Mableson } 2017[39] \\
\text { Mwingira } 2017[35] \\
\text { Martindale } 2018[37]\end{array}$ \\
\hline Nu-case & - & - & + & + & $\begin{array}{l}\text { - } \quad \text { Heterogeneity of linguistic cultural backgrounds } \\
\text { - } \quad \text { Potential legal issues } \\
\text { - } \quad \text { Network signal coverage } \\
\text { - } \quad \text { Imagility and battery life } \\
\text { - } \quad \text { Empowerment of local health workers }\end{array}$ & Luz 2015 [40] \\
\hline SkinApp & - & - & + & + & $\begin{array}{l}\text { - Technical requirements } \\
\text { - } \quad \text { Cost } \\
\text { - Need of context-specific adaptation (language, } \\
\text { culture, and epidemiological situation) }\end{array}$ & Mieras 2018 [10] \\
\hline WhatsApp & - & - & + & - & $\begin{array}{l}\text { - Data privacy } \\
\text { - } \quad \text { Highly time consuming } \\
\text { - } \quad \text { sork required on mentor and mentee relation- } \\
\text { - } \quad \text { Internet connection requirement }\end{array}$ & Akoko 2019 [42] \\
\hline
\end{tabular}

${ }^{\mathrm{a}} \mathrm{HCP}$ : health care professional.

Five interventions were conducted through apps and cloud servers. One utilized the commercially available WhatsApp app [42], and the others used either very simple ad-hoc developed apps able to collect data and send to a server [32] or apps that included a wider range of elements and took a more comprehensive approach, for example, Guaral App [38], $\mathrm{Nu}$-case [40], and SkinApp [10]. Only Guaral App and SkinApp are available from the app stores (Table 3 ). 
Table 3. Characteristics of apps for skin neglected tropical diseases available from app stores in alphabetical order.

\begin{tabular}{|c|c|c|c|c|}
\hline $\begin{array}{l}\text { Name } \\
\text { (date last updated) }\end{array}$ & $\begin{array}{l}\text { Marketplace } \\
\text { (number of } \\
\text { downloads) }\end{array}$ & Objective & Target user & Disease \\
\hline $\begin{array}{l}\text { EndNTDs App } \\
\text { (October 2017) }\end{array}$ & $\begin{array}{l}\text { Google Play } \\
\text { Store }(+100)\end{array}$ & $\begin{array}{l}\text { To create a community of champions who are at the } \\
\text { forefront in the fight against NTDs }{ }^{\mathrm{a}} \text { in Zimbabwe, } \\
\text { Africa, and globally }\end{array}$ & $\mathrm{HCP}^{\mathrm{b}}$ & $\begin{array}{l}\text { NTDs (including skin } \\
\text { NTDs) }\end{array}$ \\
\hline $\begin{array}{l}\text { Guaral RPC } \\
\text { (February 2020) }\end{array}$ & $\begin{array}{l}\text { Google Play } \\
\text { Store }(+10)\end{array}$ & To improve early diagnosis & $\mathrm{HCP}$ & $\mathrm{CL}^{\mathrm{c}}$ \\
\hline $\begin{array}{l}\text { Lepra Reaction Basic manage- } \\
\text { ment guide } \\
\text { (October 2017) }\end{array}$ & $\begin{array}{l}\text { Google Play } \\
\text { Store }(+100)\end{array}$ & $\begin{array}{l}\text { To conduct classification and quantification of severity } \\
\text { to plan for appropriate clinical management }\end{array}$ & $\mathrm{HCP}$ & Leprosy \\
\hline $\begin{array}{l}\text { LymEX } \\
\text { (July } 2019 \text { Google Play Store } \\
\text { and January } 2020 \text { Apple App } \\
\text { Store) }\end{array}$ & $\begin{array}{l}\text { Google Play } \\
\text { Store }(+50) \\
\text { Apple App } \\
\text { Store }\end{array}$ & To improve self-care & Patients & Lymphoedema \\
\hline $\begin{array}{l}\text { LymVol } \\
\text { (September 2019) }\end{array}$ & $\begin{array}{l}\text { Google Play } \\
\text { Store }(+10)\end{array}$ & $\begin{array}{l}\text { To measure and calculate limb volume of those affected } \\
\text { by edema }\end{array}$ & $\mathrm{HCP}$ & Lymphoedema \\
\hline $\begin{array}{l}\text { Recognize Hydrocele disease } \\
\text { (September 2019) }\end{array}$ & $\begin{array}{l}\text { Google Play } \\
\text { Store }(+500)\end{array}$ & $\begin{array}{l}\text { To improve information about causes, treatment, and } \\
\text { complications }\end{array}$ & Patients & Hydrocele \\
\hline $\begin{array}{l}\text { Recognize scabies } \\
\text { (September 2019) }\end{array}$ & $\begin{array}{l}\text { Google Play } \\
\text { Store }(+100)\end{array}$ & To improve knowledge about the disease & Patients & Scabies \\
\hline $\begin{array}{l}\text { Scabies disease } \\
\text { (December 2017) }\end{array}$ & $\begin{array}{l}\text { Google Play } \\
\text { Store }(+500)\end{array}$ & To improve clinical management of the disease & $\mathrm{HCP}$ & Scabies \\
\hline $\begin{array}{l}\text { Scabies Disease: Treatment } \\
\text { (October 2019) }\end{array}$ & $\begin{array}{l}\text { Google Play } \\
\text { Store }(+100)\end{array}$ & To manage symptoms and treatment & Patients & Scabies \\
\hline $\begin{array}{l}\text { SkinApp } \\
\text { (October 2019) }\end{array}$ & $\begin{array}{l}\text { Google Play } \\
\text { Store }(+10) \\
\text { Apple App } \\
\text { Store }\end{array}$ & $\begin{array}{l}\text { To act as a diagnostic tool and source of information on } \\
\text { signs, symptoms, and therapy }\end{array}$ & $\mathrm{HCP}$ & $\begin{array}{l}\text { NTD- and HIV-relat- } \\
\text { ed skin diseases }\end{array}$ \\
\hline $\begin{array}{l}\text { Skin NTDs App } \\
\text { (July 2020) }\end{array}$ & $\begin{array}{l}\text { Google Play } \\
\text { Store }(+60) \\
\text { Apple App } \\
\text { Store }\end{array}$ & $\begin{array}{l}\text { To diagnose and identify signs and symptoms of sNTDs } \\
\text { through their visible characteristics }\end{array}$ & $\mathrm{HCP}$ & Skin NTDs \\
\hline $\begin{array}{l}\text { Task Force Tropical Data } \\
\text { (August 2016) }\end{array}$ & $\begin{array}{l}\text { Google Play } \\
\text { Store }(+1000)\end{array}$ & To collect data & $\begin{array}{l}\text { Task Force for } \\
\text { Global Health } \\
\text { members }\end{array}$ & $\begin{array}{l}\text { NTDs (including skin } \\
\text { NTDs) }\end{array}$ \\
\hline $\begin{array}{l}\text { Tropical Diseases } \\
\text { (August 2019) }\end{array}$ & $\begin{array}{l}\text { Google Play } \\
\text { Store }(+1000)\end{array}$ & $\begin{array}{l}\text { To provide a detailed overview of the etiology, patho- } \\
\text { physiology, epidemiology, diagnosis, and treatment of } \\
\text { tropical diseases }\end{array}$ & $\mathrm{HCP}$ & $\begin{array}{l}\text { TDs }{ }^{\mathrm{d}} \text { including CL, } \\
\mathrm{BU}^{\mathrm{e}} \text {, and leprosy }\end{array}$ \\
\hline $\begin{array}{l}\text { Tropical Diseases } \\
\text { (December 2019) }\end{array}$ & $\begin{array}{l}\text { Google Play } \\
\text { Store }(+100)\end{array}$ & $\begin{array}{l}\text { To provide a detailed overview of the cause, diagnosis, } \\
\text { prognosis, risk factors, prevention, and treatment of the } \\
\text { stated diseases }\end{array}$ & $\mathrm{HCP}$ & $\begin{array}{l}\text { TDs including CL, } \\
\text { BU, and leprosy }\end{array}$ \\
\hline $\begin{array}{l}\text { WIDP } \\
\text { (January 2020) }\end{array}$ & $\begin{array}{l}\text { Google Play } \\
\text { Store }(+10)\end{array}$ & To control the epidemiological situation & $\begin{array}{l}\mathrm{HCP} \text { and } \mathrm{WHO}^{\mathrm{f}} \\
\text { staff }\end{array}$ & Yaws and $\mathrm{BU}$ \\
\hline
\end{tabular}

${ }^{\mathrm{a}}$ NTD: neglected tropical disease.

${ }^{\mathrm{b}} \mathrm{HCP}$ : health care professional.

${ }^{\mathrm{c}} \mathrm{CL}$ : cutaneous leishmaniasis.

${ }^{\mathrm{d}} \mathrm{TD}$ : tropical disease.

${ }^{\mathrm{e}} \mathrm{BU}$ : Buruli ulcer.

${ }^{\mathrm{f}}$ WHO: World Health Organization.

Results showed that mHealth strategies do achieve their primary and app interventions face (1) technological, (2) legal, (3) final goals, but many challenges require consideration. Both SMS user, and (4) organizational issues (Table 2) as follows: 
- The main technological issues are poor network coverage or no internet connection in specific areas, batteries running out of charge, poor data and image quality, and lack of technical requirements.

- Legal issues, including data privacy and ownership, are important concerns that must be resolved.

- Final users can be health care professionals, patients, and citizens, who all require adequate digital literacy and to feel more empowered.

- Final users need to perceive the technology as possessing a high level of usefulness and ease of use.

- The organizational challenges most often identified relate to resources (cost, time, and availability of smartphones), implementation planning, cultural and language barriers, and the need for user features to be fully completed when implementing comprehensive mHealth strategies.

mHealth interventions using apps also involve extra challenges in the development period, such as consideration of the sociotechnical context, and the need for human-centered and iterative design to improve usability, feasibility, and user experience.

\section{Selection of Apps}

Fifteen apps were identified in the Google Play Store, with three of these also available in the Apple App Store. Most of the apps $(11 / 15,73 \%)$ were targeted at health care professionals, with only four targeted at patients. The number of app downloads can be taken as a proxy indicator of use. However, while Google Play Store comprehensively provides this information, the Apple App Store provides it to the app developer only. Google Play Store data revealed that only two of the apps had more than 1000 downloads. These were Task Force Tropical Data, which is mainly used to collect data, and Tropical Diseases, which focuses on various tropical diseases but only provides information on three sNTDs (Buruli ulcer, cutaneous leishmaniasis, and leprosy). Most of the apps have recently been updated or uploaded, with only four not updated over the last 12 months. The apps addressed scabies $(3 / 15,20 \%)$, lymphatic filariasis $(3 / 15,20 \%)$, cutaneous leishmaniasis $(1 / 15,7 \%)$, leprosy $(1 / 15,7 \%)$, yaws and Buruli ulcer $(1 / 15,7 \%)$, tropical diseases including more than one sNTDs $(3 / 15,20 \%)$, and NTDs including sNTDs $(2 / 15,13 \%)$. Only $1(7 \%)$ app was related to the clinical management of sNTDs (Table 3 ).

\section{Discussion}

\section{Principal Findings}

We reviewed the literature on the evidence for mobile apps and have providing a set of recommendations for the future development and implementation of such tools. Our review pinpointed 13 studies dealing with mobile health interventions for managing sNTDs in LMICs. A descriptive evidence synthesis showed that most of the studies had a high or moderate risk of bias according to SIGN quality criteria, and only three were considered to have a low risk of bias. Only two studies focused on sNTDs other than lymphatic filariasis, one focused on cutaneous leishmaniasis, and one focused on leprosy.
In addition, eight different mHealth interventions were identified, of which three were based on texting by SMS and five were conducted through apps and cloud servers. Moreover, we acknowledged 15 apps available in app stores. Most of the apps $(11 / 15,73 \%)$ were targeted at health care professionals, with only four targeted at patients. Results showed that mHealth strategies do achieve their primary goals, but many challenges require consideration. All mHealth interventions face technological, legal, final user, and organizational issues.

The disease most targeted in mHealth interventions for sNTDs is lymphatic filariasis. The Global Program to Eliminate Lymphatic Filariasis as a public health problem was launched in 2000, and the global elimination goal of 2020 established in 2012 was probably a key factor in driving the Ministry of Health, the WHO, and implementing partners to scale up surveillance and morbidity management activities for this disease. There is an urgent need for a rapid and adaptable tool to gather patient estimates in order for national programs to appropriately forecast, plan, and deliver a basic package of care to those suffering from the disabling and debilitating clinical manifestations of lymphatic filariasis in an affordable manner [39]. Three of the apps on the market address scabies, but as no trials have been identified, there is a lack of existing evidence about mHealth interventions to manage this disease. Other sNTDs, such as cutaneous leishmaniasis and leprosy, have been managed with pilot interventions through mHealth strategies. It is interesting to note that a number of trials and apps are focused on an integrated approach to the various sNTDs. The most recent app developed by WHO aims to provide support for diagnosis by using an automated algorithm to provide potential conditions from the selection of SNTD signs and symptoms.

The provision of health care services to populations in remote regions, often dispersed over large geographical areas, has long been considered a challenging issue from the perspective of health systems management [44]. Telemedicine approaches have been employed in some places for remote assistance for diagnosis and treatment, with a degree of success [45], but neglected diseases, and therefore sNTDs, require more than the usual functionality of telemedicine systems [40]. mHealth interventions in high-income countries are focused on apps, but as sNTDs mainly affect populations with low resources in LMICs, most of the interventions tested so far were based on SMS text messaging, and only a few app strategies have been attempted. Using text messages to change patient behavior and achieve targeted health outcomes also requires a comprehensive strategy but demands fewer resources. Studies in Indonesia have addressed the apparent difficulties in leprosy control programs and how a health information system could assist, and concluded that SMS text messaging and web-based applications could be good strategies for implementing continuous monitoring and recording of patients [43]. The advantage of SMS is "internet-less" connectivity, and it allows fast transmission of surveillance data, whereas store-and-forward teledermatology represents an ideal approach to assess skin lesions and provide long-distance support to individual diagnosis because images are shared and can be assessed. The cost-effectiveness of store-and-forward teledermatology increases when patients are 
required to travel farther distances to access dermatology services. SMS and store-and-forward teledermatology are complementary and contribute to improve the epidemiological and clinical management of sNTDs.

By bringing essential central health services closer to peripheral areas, innovative technological methods like telemedicine, mHealth apps, and SMS text messages can help to bridge the gap between the burden of skin diseases and the lack of capable staff in resource-poor settings [10]. mHealth interventions enable rapid data collection, easy monitoring and supervision of data reporting, better management of sNTDs, and efficient provision of necessary care. They also appear to be lower cost strategies than paper-based forms that require more human and financial resources. The four studies that conducted cost analyses [33,35-37] did not have sufficient robust data, but all concluded that mHealth interventions are less costly than paper-based epidemiological mapping. By managing and taking full ownership of data and the implementation process, local teams will be empowered, and the establishment of valuable data management and health surveillance capacities within the local team and at the country level will empower national health systems [35].

\section{Recommendations Based on the Existing Evidence}

Based on evidence presented in the studies identified in this systematic review, the following eight recommendations will enable sNTD mHealth-based interventions to move forward from innovation to implementation:

1. No one should be left behind. Patients from all regions must be targeted to benefit from the proposed interventions. This requires translation of the tools into several languages. At least into Portuguese and Spanish in the Americas, and English, French, and Portuguese in Africa.

2. Users must be empowered. Final users of the interventions (health care professionals and/or patients) should receive sufficient training to improve their digital literacy and make appropriate use of the tools.

3. Complexity must be addressed. The adoption of digital health technology is a complicated process that needs to be thoroughly considered before and during implementation.

4. Utility and simplicity must be perceived. Health care professionals, patients, and healthy citizens should grasp the utility and user friendliness of the proposed technology, in order for these two elements to become enablers and not barriers.

5. Technological requirements must be considered from the very outset. The availability of smartphones and potential problems with electricity or internet networks must be addressed as part of a comprehensive strategy with a specific aim.

6. A long-term mHealth platform must be put in place. The success of an mHealth intervention depends on the existence of an mHealth platform to not only facilitate adoption of the tool, but also guarantee sustained effective use.

7. Two-tiered processes are required for improvement. In the first stages of implementing an mHealth intervention, it is important to have two-tiered processes to refine and optimize the process in an iterative way.

8. The tool must respond to needs. Interventions are embedded in a specific health service; therefore, additional tools must be considered according to the need.

\section{Limitations}

One of the main limitations of this review is publication bias. References from other sources, such as conferences and meetings, have not been included. Although the number of scientific journals that publish mHealth-related articles has increased in recent years, there is a lot of grey literature surrounding this field that we may have missed. Moreover, the heterogeneity of interventions and populations has made it difficult to synthesize results, and consequently, findings need to be considered with caution. Most studies in this review were conducted in East Africa, and the total number of countries represents $9 \%$ of those with NTDs worldwide. This limits the extrapolation of the results.

\section{Conclusions}

The potential for mHealth interventions to improve the epidemiological and clinical management of sNTDs is yet to be reached. There are very few good quality studies and tools, and most of these have methodological limitations, leaving considerable room for improvement. The majority of sNTDs are not addressed by any mHealth tool. This research has enabled us to identify eight recommendations for the future development and implementation of mHealth interventions for managing sNTDs in LMICs.

\section{Acknowledgments}

All authors contributed equally. The authors thank GOC Networking and Marta Pulido, MD, PhD, for editing the manuscript and editorial assistance.

\section{Conflicts of Interest}

None declared.

\section{Multimedia Appendix 1}

Search strategy.

[DOCX File, 55 KB-Multimedia Appendix 1]

\section{References}


1. Control of Neglected Tropical Diseases. World Health Organization. URL: http://www.who.int/neglected_diseases/diseases/ en/\# [accessed 2020-12-14]

2. Working to overcome the global impact of neglected tropical diseases: First WHO report on Neglected Tropical Diseases. World Health Organization. URL: https://www.who.int/neglected_diseases/resources/9789241564090/en/ [accessed 2020-12-14]

3. Verrest L, Dorlo TPC. Lack of Clinical Pharmacokinetic Studies to Optimize the Treatment of Neglected Tropical Diseases: A Systematic Review. Clin Pharmacokinet 2017 Jun 15;56(6):583-606 [FREE Full text] [doi: 10.1007/s40262-016-0467-3] [Medline: 27744580]

4. Gómez LJ, van Wijk R, van Selm L, Rivera A, Barbosa M, Parisi S, et al. Stigma, participation restriction and mental distress in patients affected by leprosy, cutaneous leishmaniasis and Chagas disease: a pilot study in two co-endemic regions of eastern Colombia. Trans R Soc Trop Med Hyg 2020 Jul 01;114(7):476-482 [FREE Full text] [doi: 10.1093/trstmh/trz132] [Medline: 32052043]

5. Pires M, Wright B, Kaye PM, da Conceição V, Churchill RC. The impact of leishmaniasis on mental health and psychosocial well-being: A systematic review. PLoS One 2019 Oct 17;14(10):e0223313 [FREE Full text] [doi: 10.1371/journal.pone.0223313] [Medline: 31622369]

6. van 't Noordende AT, Aycheh MW, Schippers A. The impact of leprosy, podoconiosis and lymphatic filariasis on family quality of life: A qualitative study in Northwest Ethiopia. PLoS Negl Trop Dis 2020 Mar;14(3):e0008173 [FREE Full text] [doi: 10.1371/journal.pntd.0008173] [Medline: 32155158]

7. Engelman D, Fuller LC, Solomon AW, McCarthy JS, Hay RJ, Lammie PJ, et al. Opportunities for Integrated Control of Neglected Tropical Diseases That Affect the Skin. Trends Parasitol 2016 Nov;32(11):843-854. [doi: 10.1016/j.pt.2016.08.005] [Medline: 27638231]

8. Mitjà O, Marks M, Bertran L, Kollie K, Argaw D, Fahal AH, et al. Integrated Control and Management of Neglected Tropical Skin Diseases. PLoS Negl Trop Dis 2017 Jan;11(1):e0005136 [FREE Full text] [doi: 10.1371/journal.pntd.0005136] [Medline: 28103250]

9. Yotsu RR, Kouadio K, Vagamon B, N'guessan K, Akpa AJ, Yao A, et al. Skin disease prevalence study in schoolchildren in rural Côte d'Ivoire: Implications for integration of neglected skin diseases (skin NTDs). PLoS Negl Trop Dis 2018 May 17;12(5):e0006489 [FREE Full text] [doi: 10.1371/journal.pntd.0006489] [Medline: 29771976]

10. Mieras L, Taal A, Post E, Ndeve A, van Hees C. The Development of a Mobile Application to Support Peripheral Health Workers to Diagnose and Treat People with Skin Diseases in Resource-Poor Settings. Trop Med Infect Dis 2018 Sep 15;3(3):102 [FREE Full text] [doi: 10.3390/tropicalmed3030102] [Medline: 30274498]

11. Rat C, Hild S, Rault Sérandour J, Gaultier A, Quereux G, Dreno B, et al. Use of Smartphones for Early Detection of Melanoma: Systematic Review. J Med Internet Res 2018 Apr 13;20(4):e135 [FREE Full text] [doi: 10.2196/jmir.9392] [Medline: 29653918]

12. Patterson V. Telemedicine for epilepsy support in resource-poor settings. Front Public Health 2014 Aug 21;2:120 [FREE Full text] [doi: 10.3389/fpubh.2014.00120] [Medline: 25191650]

13. Wootton R, Geissbuhler A, Jethwani K, Kovarik C, Person D, Vladzymyrskyy A, et al. Long-running telemedicine networks delivering humanitarian services: experience, performance and scientific output. Bull World Health Org 2012 May 01;90(5):341-347. [doi: 10.2471/blt.11.099143]

14. Delaigue S, Morand J, Olson D, Wootton R, Bonnardot L. Teledermatology in Low-Resource Settings: The MSF Experience with a Multilingual Tele-Expertise Platform. Front Public Health 2014;2:233 [FREE Full text] [doi:

10.3389/fpubh.2014.00233] [Medline: 25453029]

15. Surovi N, Kiber A, Kashem A, Babi KN. Study and development of algorithm of different skin diseases analysis using image processing method. Asian J Biomed Pharmaceut Sci 2017;7(60) [FREE Full text]

16. Yadav N, Kumar V, Shrivastava U. Skin Diseases Detection Models using Image Processing: A Survey. IJCA 2016 Mar 17;137(12):34-39. [doi: 10.5120/ijca2016909001]

17. Garcia-Vidal C, Sanjuan G, Puerta-Alcalde P, Moreno-García E, Soriano A. Artificial intelligence to support clinical decision-making processes. EBioMedicine 2019 Aug;46:27-29 [FREE Full text] [doi: 10.1016/j.ebiom.2019.07.019] [Medline: $\underline{\text { 31303500] }}$

18. Peiffer-Smadja N, Dellière S, Rodriguez C, Birgand G, Lescure F, Fourati S, et al. Machine learning in the clinical microbiology laboratory: has the time come for routine practice? Clin Microbiol Infect 2020 Oct 12;26(10):1300-1309. [doi: 10.1016/j.cmi.2020.02.006] [Medline: 32061795]

19. Ryu S. Book Review: mHealth: New Horizons for Health through Mobile Technologies: Based on the Findings of the Second Global Survey on eHealth (Global Observatory for eHealth Series, Volume 3). Healthc Inform Res 2012;18(3):231. [doi: 10.4258/hir.2012.18.3.231]

20. Puigdomenech Puig E, Robles N, Saigí-Rubió F, Zamora A, Moharra M, Paluzie G, et al. Assessment of the Efficacy, Safety, and Effectiveness of Weight Control and Obesity Management Mobile Health Interventions: Systematic Review. JMIR Mhealth Uhealth 2019 Oct 25;7(10):e12612 [FREE Full text] [doi: 10.2196/12612] [Medline: $\underline{31654566}$ ] 
21. Wood CS, Thomas MR, Budd J, Mashamba-Thompson TP, Herbst K, Pillay D, et al. Taking connected mobile-health diagnostics of infectious diseases to the field. Nature 2019 Feb;566(7745):467-474 [FREE Full text] [doi: 10.1038/s41586-019-0956-2] [Medline: $\underline{30814711]}$

22. 39+ smartphone statistics you should know in 2020. Review 42. 2020. URL: https://review42.com/smartphone-statistics/ [accessed 2020-12-14]

23. Rizvi SMH, Schopf T, Sangha A, Ulvin K, Gjersvik P. Teledermatology in Norway using a mobile phone app. PLoS One 2020 Apr 27;15(4):e0232131 [FREE Full text] [doi: 10.1371/journal.pone.0232131] [Medline: 32339203]

24. Chan S, Reddy V, Myers B, Thibodeaux Q, Brownstone N, Liao W. Machine Learning in Dermatology: Current Applications, Opportunities, and Limitations. Dermatol Ther (Heidelb) 2020 Jun;10(3):365-386 [FREE Full text] [doi: 10.1007/s13555-020-00372-0] [Medline: 32253623]

25. Kho J, Gillespie N, Horsham C, Snoswell C, Vagenas D, Soyer HP, et al. Skin Doctor Consultations Using Mobile Teledermoscopy: Exploring Virtual Care Business Models. Telemed J E Health 2020 Nov 14;26(11):1406-1413. [doi: 10.1089/tmj.2019.0228] [Medline: 32058835]

26. mHealth Economics 2017 - Current Status and Future Trends in Mobile Health. Research 2 Guidance. 2017. URL: https:/ /research2guidance.com/product/mhealth-economics-2017-current-status-and-future-trends-in-mobile-health/ [accessed 2020-12-14]

27. Flaten HK, St Claire C, Schlager E, Dunnick CA, Dellavalle RP. Growth of mobile applications in dermatology - 2017 update. Dermatol Online J 2018 Feb 15;24(2) [FREE Full text] [Medline: 29630159]

28. Freeman K, Dinnes J, Chuchu N, Takwoingi Y, Bayliss SE, Matin RN, et al. Algorithm based smartphone apps to assess risk of skin cancer in adults: systematic review of diagnostic accuracy studies. BMJ 2020 Feb 10;368:m127 [FREE Full text] [doi: 10.1136/bmj.m127] [Medline: $\underline{\text { 32041693] }}$

29. Charbonneau DH, Hightower S, Katz A, Zhang K, Abrams J, Senft N, et al. Smartphone apps for cancer: A content analysis of the digital health marketplace. Digit Health 2020 Feb 11;6:2055207620905413 [FREE Full text] [doi:

10.1177/2055207620905413] [Medline: 32110428]

30. Moher D, Liberati A, Tetzlaff J, Altman DG, PRISMA Group. Preferred reporting items for systematic reviews and meta-analyses: the PRISMA statement. PLoS Med 2009 Jul 21;6(7):e1000097 [FREE Full text] [doi:

10.1371/journal.pmed.1000097] [Medline: 19621072]

31. Scottish Intercollegiate Guidelines Network. SIGN 50: A guideline developer's handbook. Edinburgh, Scotland: Scottish Intercollegiate Guidelines Network (SIGN); 2008.

32. Sime H, Deribe K, Assefa A, Newport MJ, Enquselassie F, Gebretsadik A, et al. Integrated mapping of lymphatic filariasis and podoconiosis: lessons learnt from Ethiopia. Parasit Vectors 2014 Aug 27;7:397 [FREE Full text] [doi: 10.1186/1756-3305-7-397] [Medline: 25164687]

33. Stanton MC, Mkwanda SZ, Debrah AY, Batsa L, Biritwum N, Hoerauf A, et al. Developing a community-led SMS reporting tool for the rapid assessment of lymphatic filariasis morbidity burden: case studies from Malawi and Ghana. BMC Infect Dis 2015 May 16;15:214 [FREE Full text] [doi: 10.1186/s12879-015-0946-4] [Medline: 25981497]

34. Mwingira UJ, Downs P, Uisso C, Chikawe M, Sauvage-Mar M, Malecela MN, et al. Applying a mobile survey tool for assessing lymphatic filariasis morbidity in Mtwara Municipal Council of Tanzania. Mhealth 2017 Mar 15;3:8-8 [FREE Full text] [doi: 10.21037/mhealth.2017.03.01] [Medline: 28567405]

35. Mwingira U, Chikawe M, Mandara WL, Mableson HE, Uisso C, Mremi I, et al. Lymphatic filariasis patient identification in a large urban area of Tanzania: An application of a community-led mHealth system. PLoS Negl Trop Dis 2017 Jul 14;11(7):e0005748 [FREE Full text] [doi: 10.1371/journal.pntd.0005748] [Medline: 28708825]

36. Karim MJ, Haq R, Mableson HE, Sultan Mahmood ASM, Rahman M, Chowdhury SM, et al. Developing the first national database and map of lymphatic filariasis clinical cases in Bangladesh: Another step closer to the elimination goals. PLoS Negl Trop Dis 2019 Jul;13(7):e0007542 [FREE Full text] [doi: 10.1371/journal.pntd.0007542] [Medline: $\underline{31306409]}$

37. Martindale S, Mableson HE, Kebede B, Kiros FH, Tamiru A, Mengistu B, et al. A comparison between paper-based and $\mathrm{m}$-Health tools for collating and reporting clinical cases of lymphatic filariasis and podoconiosis in Ethiopia. Mhealth 2018 Oct;4:49-49 [FREE Full text] [doi: 10.21037/mhealth.2018.09.12] [Medline: 30505847]

38. Navarro A, Rubiano L, Arango JD, Rojas CA, Alexander N, Saravia NG, et al. Developing mobile health applications for neglected tropical disease research. PLoS Negl Trop Dis 2018 Nov 1;12(11):e0006791 [FREE Full text] [doi: 10.1371/journal.pntd.0006791] [Medline: 30383809]

39. Mableson HE, Martindale S, Stanton MC, Mackenzie C, Kelly-Hope LA. Community-based field implementation scenarios of a short message service reporting tool for lymphatic filariasis case estimates in Africa and Asia. Mhealth 2017 Jul 21;3:28-28 [FREE Full text] [doi: 10.21037/mhealth.2017.06.06] [Medline: 28828375]

40. Luz S, Masoodian M, Cesario M. Disease surveillance and patient care in remote regions: an exploratory study of collaboration among health-care professionals in Amazonia. Behaviour \& Information Technology 2014 Jan 13;34(6):548-565. [doi: 10.1080/0144929x.2013.853836]

41. Pedram B, Pasquetto V, Drame PM, Ji Y, Gonzalez-Moa MJ, Baldwin RK, et al. A novel rapid test for detecting antibody responses to Loa loa infections. PLoS Negl Trop Dis 2017 Jul 27;11(7):e0005741 [FREE Full text] [doi:

10.1371/journal.pntd.0005741] [Medline: 28749939] 
42. Akoko L, Mwanga A, Chikawe M, Lutainulwa E, Ngoma D, Nshalla A, et al. Supervision and support in surgical practice using mobile platform: a case of mass hydrocele surgeries in remote regions. Mhealth 2019 Sep;5:41-41 [FREE Full text] [doi: $10.21037 /$ mhealth.2019.09.05] [Medline: $\underline{31620468]}$

43. Rachmani E, Hsu C, Chang PWS, Jumanto J, Fuad A, Ningrum DNA, et al. Encouraging On-Time Completion of Leprosy Patients Treatment: Implementing E-Leprosy Framework to Primary Health Care in Indonesia. Asia Pac J Public Health 2019 May;31(4):296-305. [doi: 10.1177/1010539519847355] [Medline: 31104477]

44. Dussault G, Franceschini MC. Not enough there, too many here: understanding geographical imbalances in the distribution of the health workforce. Hum Resour Health 2006 May 27;4:12 [FREE Full text] [doi: 10.1186/1478-4491-4-12] [Medline: 16729892]

45. Hailey D, Roine R, Ohinmaa A. Systematic review of evidence for the benefits of telemedicine. J Telemed Telecare 2002 Dec 02;8 Suppl 1(1_suppl):1-30. [doi: 10.1258/1357633021937604] [Medline: 12020415]

\author{
Abbreviations \\ LMIC: low- and middle-income country \\ mHealth: mobile health \\ NTD: neglected tropical disease \\ SIGN: Scottish Intercollegiate Guidelines Network \\ SNTD: skin neglected tropical disease \\ WHO: World Health Organization
}

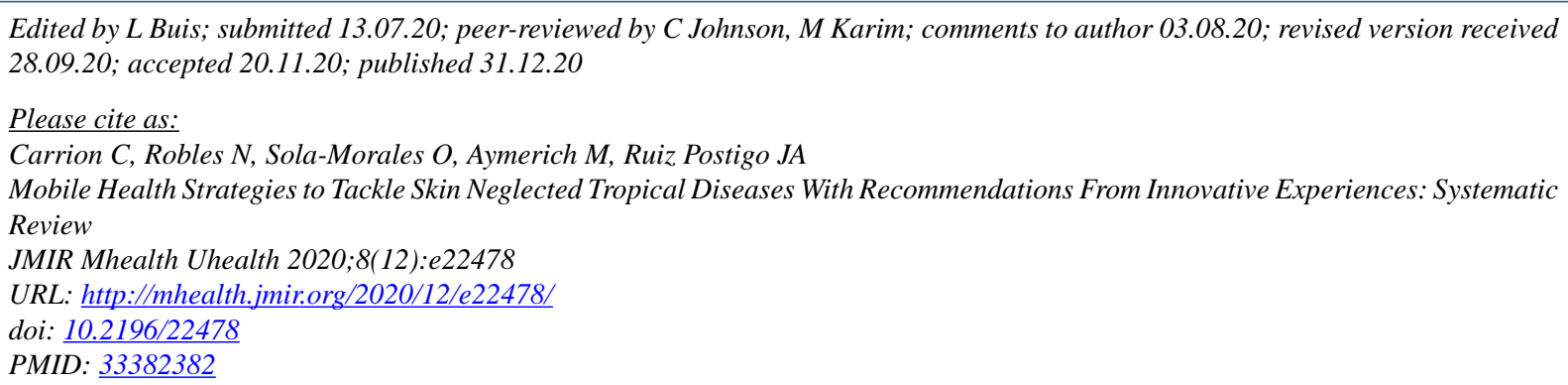

(C) Carme Carrion, Noemí Robles, Oriol Sola-Morales, Marta Aymerich, Jose Antonio Ruiz Postigo. Originally published in JMIR mHealth and uHealth (http://mhealth.jmir.org), 31.12.2020. This is an open-access article distributed under the terms of the Creative Commons Attribution License (https://creativecommons.org/licenses/by/4.0/), which permits unrestricted use, distribution, and reproduction in any medium, provided the original work, first published in JMIR mHealth and uHealth, is properly cited. The complete bibliographic information, a link to the original publication on http://mhealth.jmir.org/, as well as this copyright and license information must be included. 